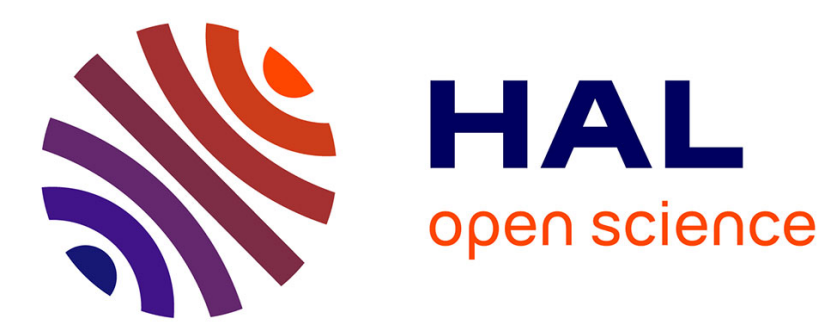

\title{
Output feedback control for quadratic systems: A Lyapunov function approach
}

Eduardo S Tognetti, Marc Jungers, Taís R Calliero

\section{To cite this version:}

Eduardo S Tognetti, Marc Jungers, Taís R Calliero. Output feedback control for quadratic systems: A Lyapunov function approach. International Journal of Robust and Nonlinear Control, 2021, 31 (17), pp.8373-8389. 10.1002/rnc.5435 . hal-03292389

\section{HAL Id: hal-03292389 \\ https://hal.science/hal-03292389}

Submitted on 20 Jul 2021

HAL is a multi-disciplinary open access archive for the deposit and dissemination of scientific research documents, whether they are published or not. The documents may come from teaching and research institutions in France or abroad, or from public or private research centers.
L'archive ouverte pluridisciplinaire HAL, est destinée au dépôt et à la diffusion de documents scientifiques de niveau recherche, publiés ou non, émanant des établissements d'enseignement et de recherche français ou étrangers, des laboratoires publics ou privés. 
DOI: $\mathrm{xxx} / \mathrm{xxxx}$

\title{
RESEARCH ARTICLE
}

\section{Output feedback control for quadratic systems: a Lyapunov function approach}

\author{
Eduardo S. Tognetti*1 | Marc Jungers ${ }^{2}$ | Taís R. Calliero ${ }^{3}$
}

\footnotetext{
${ }^{1}$ Department of Electrical Engineering, University of Brasilia - UnB, 70910-900, Brasília, DF, Brazil

${ }^{2}$ Université de Lorraine, CNRS, CRAN, F-54000 Nancy, France

${ }^{3}$ Faculty of Technology Gama, University of Brasilia - UnB, 70910-900, Brasília, DF, Brazil
}

\section{Correspondence}

*Corresponding author name. Eduardo S.

Tognetti. Email: estognetti@ene.unb.br

\section{Funding Information}

This research was supported by the project ANR HANDY, ANR-18-CE40-0010 (M. Jungers) and the Brazilian agency FAP-DF Grant 00193.00002099/2018-65 (E. S. Tognetti).

\begin{abstract}
Summary
This paper deals with the design of quadratic and linear dynamic output feedback controllers for quadratic systems to ensure, on the one hand, local exponential stability of the origin in the closed-loop form and, on the other hand, to increase an estimate of the basin of attraction of the origin as large as possible. The introduction of a quadratic term in the controller permits to consider a controller in the same class of dynamics as the studied system. Here, our approach is to consider a Lyapunov function with respect to the extended state gathering the states of the system and the controller. The induced nonlinear inequalities are treated thanks to an auxiliary vector repeating adequately the extended state and by combining distinct linearization techniques to finally obtain Linear Matrix Inequalities (LMIs). For comparison purposes, we provide also an other approach characterizing the extended state as belonging to a polytope in the state space. The results are illustrated by numerical examples.
\end{abstract}

\section{KEYWORDS:}

Quadratic systems, Output feedback control, Lyapunov function approach

\section{1 | INTRODUCTION}

Dynamics that are characterized by quadratic forms with respect to the state are called quadratic systems $\mathbf{1}^{1}$. Such a system is of particular interest in the control community for several motivations. First of all, they model physical systems as for instance attitude control ${ }^{1}$, predator-prey Lotka-Volterra equations ${ }^{2.3}$, or behaviors of tumors in health ${ }^{4}$ to cite a few. More generically, quadratic systems can be viewed as the easiest nonlinear Taylor expansion, with respect to the state, of a nonlinear dynamics and the most simple nonlinear state polynomial systems ${ }^{5}$. They are closely related to the class of bilinear systems because they can be formalized as the interconnection between a bilinear system and a linear state feedback ${ }^{6}$ : they thus consist in one adequate tool to study their properties in closed form. Furthermore quadratic systems present significant and hard breaks with the field of linear systems. Of course they may involve distinct equilibrium points, cyclic or chaotic behaviors (Lorenz system is a quadratic one for instance). They may admit a finite escape time, as differential Riccati equations $\stackrel{7.8}{\text {, }}$, which is not possible in the linear framework.

A large literature concerns important properties of quadratic systems for the purpose of their analysis and their control design like minimal realization $\underline{1}^{\frac{1}{9}}$ stability analysis, local stabilization ${ }^{9}$, controllability or observability $\underline{10}$. Several of them are derived by control affine systems ${ }^{\underline{9}}$, others are specific to the class of quadratic systems: as, for instance, the classification introducted by Markus 11 thanks to a new algebra multiplication leading in this algebra to a Riccati equation ${ }^{8}$.

Due to the nonlinear nature of quadratic systems, the emphasis was placed on the local aspect of stability/stabilization and consequently on the challenging estimate of the domain of attraction of the origin. Generally the approaches are based on a local 
(quadratic) Lyapunov function and its resulting (ellipsoidal) level sets 12,13 , but the induced conditions are handled with distinct frameworks, among them: state-dependent Linear Matrix Inequalities (LMIs) solved via a double non-convex optimization problem ${ }^{14}$; increase the size of a polytope in the state space that contains the origin and belongs to its domain of attraction by taking advantages of contributions for quasi-Linear Parameter-Varying (LPV) systems $\stackrel{15.16}{ }$; state-dependent linear matrix inequalities treated with an elegant parametrization of the points of an ellipsoid and standard LMIs formulations $\frac{17}{}{ }^{17}$; sum of squares $\frac{18}{}$.

It should be underlined that, for quadratic systems, an obvious majority of controller designs are (nonlinear) state feedbacks. Only few works cope with output feedbacks, which are more difficult 19 . We can cite, not exhaustively, the contribution for control-affine systems ${ }^{20}$, the observer-based approach ${ }^{21}$ or the recent use of hybrid techniques for hybrid output feedbacks ${ }^{22}$. Furthermore, it has been noticed that extending the class of controllers to the nonlinear ones (or at least quadratic ones) may be beneficial, whether for state feedbacks $\frac{23,24}{}$ or output feedbacks ${ }^{21,25}$.

Our contribution, in this paper, is to provide quadratic and linear dynamic output feedback (DOF) controllers design for continuous-time quadratic systems by following two objectives: the first one is to locally exponentially stabilize the quadratic system. The second one is to provide an estimate of the basin of attraction of the origin as large as possible. Such a design faces to a main difficulty: matrix inequalities that are nonlinear with respect to the variables and also that depend on the extended state (state of the system and state of the controller). To deal with the state dependency in the design conditions, we adopted the parametrization of the states in an ellipsoid 17 introducing a compact notation by means of the Kronecker product. Classical linearization techniques fail and we propose a suitable combination of them (congruence transformation and specific choice of slack variables associated with Finsler's lemma) to obtain sufficient conditions as LMIs to be used as constraints of an optimization problem.

The paper is organized as follows. Section 2 presents the problem statement and Section 3 gathers a suitable rewriting of the quadratic systems thanks to an auxiliary vector repeating the extended state and also helpful technical lemmas. Section4 4 provides three main results: the design of a quadratic dynamic output feedback, the design of a linear one and finally a comparative result using the polytopic approach. Numerical illustrations and detailed discussions are given in Section 5 before concluding remarks in Section 6 .

Notation. The notation $\mathbb{R}^{n}, \mathbb{R}_{+}$and $\mathbb{R}^{n \times m}$ respectively denote the sets of $n$-dimensional real vectors, positive scalars, and $n \times m$-dimensional real matrices. For a matrix $A, A_{(i)}$ denotes the $i$ th row of $A, A^{T}$ and $A^{-T}$ denote the transpose of $A$ and $A^{-1}$, respectively, $\operatorname{vec}(A)$ is the vectorization operator of $A$ (that is, the vector concatenating all the columns of $A$ ) and, if $A$ is square, $\operatorname{He}\{A\}=A+A^{T}$. The notation co $\left\{M_{1}, \ldots, M_{N}\right\}$ denotes the convex hull formed by the matrices (or vectors) $M_{j}$, $j=1, \ldots, N$, and the block-diagonal matrix is denoted by $\operatorname{diag}(\cdot)$. The identity matrix of order $n$ is denoted by $I_{n}$ and the null $m \times n$ matrix is denoted by $0_{m, n}$ (or simply $I$ and 0 if no confusion arises). The symbol $\star$ denotes symmetric blocks in partitioned matrices, $\otimes$ and $\square$ stand, respectively, for the Kronecker product and for elements that have no influence on the development, and $\mathbf{1}$ is a vector whose components are all equal to one.

\section{I PROBLEM DEFINITION}

Consider the following continuous-time nonlinear system, which is quadratic in the state and affine in the input:

$$
\begin{aligned}
& \dot{x}(t)=A_{0} x(t)+A(x(t)) x(t)+B_{0} u(t)+B(x(t)) u(t), \\
& y(t)=C x(t),
\end{aligned}
$$

and where $x(t) \in \mathbb{R}^{n}$ is the state vector, $u(t) \in \mathbb{R}^{m}$ is the input signal and $y(t) \in \mathbb{R}^{r}$ is the measured output. The system matrices are $A_{0} \in \mathbb{R}^{n \times n}, B_{0} \in \mathbb{R}^{n \times m}$ and $C \in \mathbb{R}^{r \times n}$. The matrix-valued functions $A: \mathbb{R}^{n} \mapsto \mathbb{R}^{n \times n}$ and $B: \mathbb{R}^{n} \mapsto \mathbb{R}^{n \times m}$ are defined, respectively, by

$$
A(x):=\left[A_{1}^{T} x|\cdots| A_{n}^{T} x\right]^{T}, \quad B(x):=\left[B_{1}^{T} x|\cdots| B_{n}^{T} x\right]^{T},
$$

with $A_{i} \in \mathbb{R}^{n \times n}$ and $B_{i} \in \mathbb{R}^{n \times m}, i=1, \ldots, n$, constant known matrices.

The problem concerned in this paper is the local exponential stabilization of the origin of system (1), with an estimate of the basin of attraction of the origin as large as possible, via the nonlinear full-order DOF controller of the form

$$
\begin{aligned}
\dot{x}_{c}(t) & =A_{c} x_{c}(t)+L\left(x_{c}(t)\right) x_{c}(t)+B_{c} y(t)+K\left(x_{c}(t)\right) y(t), \\
u(t) & =C_{c} x_{c}(t)+D_{c} y(t),
\end{aligned}
$$


where $x_{c}(t) \in \mathbb{R}^{n}$ and matrices $A_{c} \in \mathbb{R}^{n \times n}, B_{c} \in \mathbb{R}^{n \times r}, C_{c} \in \mathbb{R}^{m \times n}, D_{c} \in \mathbb{R}^{m \times r}$ and matrix-valued functions $L(\cdot): \mathbb{R}^{n} \mapsto \mathbb{R}^{n \times n}$ and $K(\cdot): \mathbb{R}^{n} \mapsto \mathbb{R}^{n \times r}$ given by

$$
L\left(x_{c}\right)=\left[L_{1}^{T} x_{c}|\cdots| L_{n}^{T} x_{c}\right]^{T}, \quad K\left(x_{c}\right)=\left[K_{1}^{T} x_{c}|\cdots| K_{n}^{T} x_{c}\right]^{T},
$$

with $L_{i} \in \mathbb{R}^{n \times n}$ and $K_{i} \in \mathbb{R}^{n \times r}, i=1, \ldots, n$.

By defining an augmented state vector $\xi=\left[\begin{array}{ll}x^{T} & x_{c}^{T}\end{array}\right]^{T} \in \mathbb{R}^{2 n}$, the closed-loop system combining (1) and (3) is given by the following quadratic system

$$
\dot{\xi}(t)=A_{c l}(\xi(t)) \xi(t)
$$

where

$$
A_{c l}(\xi)=\left[\begin{array}{cc}
A_{0}+A(x)+\left(B_{0}+B(x)\right) D_{c} C & \left(B_{0}+B(x)\right) C_{c} \\
\left(B_{c}+K\left(x_{c}\right)\right) C & A_{c}+L\left(x_{c}\right)
\end{array}\right] .
$$

The quadratic system (5) may present several equilibrium points besides the origin or equilibrium cycles. The question of global stability of the origin is thus difficult and presents a restricted interest. Here we concentrate our study on designing a controller such that the origin is locally exponentially stable and providing an estimate of the domain of attraction as large as possible. As a matter of simplicity, we will consider the dynamic controller (3) with zero initial conditions then our interest is to maximize the estimation of the region of initial conditions $x(0)$, denoted by $S_{0} \subseteq \mathbb{R}^{n}$, such that the trajectories $\xi(t)$ converge to the origin. The problem addressed in this paper can be summarized as follows.

Problem 1. Determine a nonlinear dynamic output controller as (3) and a region $S_{0} \subseteq \mathbb{R}^{n}$, as large as possible, such that the trajectories of closed-loop system (5) starting from any initial condition $\left(x(0), x_{c}(0)\right) \in S_{0} \times\{0\}$ converge exponentially towards the origin.

Before presenting the main results in Section 4 , the following section provides preliminary tools and results.

\section{3 | PRELIMINARIES}

Observe that for any matrix $M \in \mathbb{R}^{n \times m}$ and a vector $v \in \mathbb{R}^{n}$ one can write $v^{T} M=\operatorname{vec}(M)^{T}\left(I_{n} \otimes v\right)$. Moreover, we can rewrite the product involving the states $x$ and $x_{c}$ in terms of the augmented state $\xi$, that is, $x^{T} A_{i}=\xi^{T}\left[A_{i}^{T} 0\right]^{T}$ and $x_{c}^{T} L_{i}=\xi^{T}\left[0 L_{i}^{T}\right]^{T}$ (the same involving $B_{i}$ and $K_{i}$ ). Define $\hat{\xi}_{K}:=I_{\kappa} \otimes \xi \in \mathbb{R}^{2 n \kappa \times \kappa}$, then, one has

$$
A(x)=\tilde{A} \hat{\xi}_{n}, \quad B(x)=\tilde{B} \hat{\xi}_{m}, \quad L(x)=\tilde{L} \hat{\xi}_{n}, \quad K(x)=\tilde{K} \hat{\xi}_{r},
$$

where

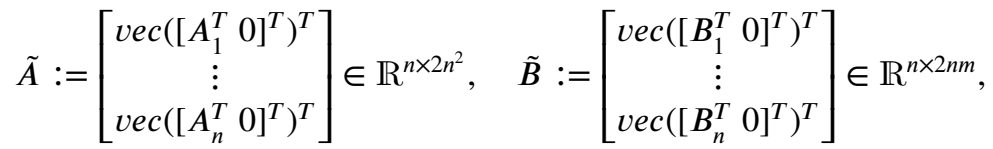

$$
\begin{aligned}
& \tilde{L}:=\left[\begin{array}{c}
\operatorname{vec}\left(\left[0 L_{1}^{T}\right]^{T}\right)^{T} \\
\vdots \\
\operatorname{vec}\left(\left[0 L_{n}^{T}\right]^{T}\right)^{T}
\end{array}\right] \in \mathbb{R}^{n \times 2 n^{2}}, \quad \tilde{K}:=\left[\begin{array}{c}
\operatorname{vec}\left(\left[0 K_{1}^{T}\right]^{T}\right)^{T} \\
\vdots \\
\operatorname{vec}\left(\left[\begin{array}{ll}
0 & K_{n}^{T}
\end{array}\right]^{T}\right)^{T}
\end{array}\right] \in \mathbb{R}^{n \times 2 n r} .
\end{aligned}
$$

One of the convenient tool considered here is the auxiliary vector concatenating enough times the extended state $\xi$. It is noteworthy that this is a crucially different technique from the one modeling the quadratic term with a multiple Kronecker product of the extended state 26,27 . The closed-loop dynamics (6) is rewritten as

$$
A_{c l}(\xi)=\left[\begin{array}{cc}
A_{0}+\tilde{A} \hat{\xi}_{n}+\left(B_{0}+\tilde{B} \hat{\xi}_{m}\right) D_{c} C & \left(B_{0}+\tilde{B} \hat{\xi}_{m}\right) C_{c} \\
\left(B_{c}+\tilde{K} \hat{\xi}_{n}\right) C & A_{c}+\tilde{L} \hat{\xi}_{r}
\end{array}\right]=A_{c l 0}+A_{c l, \xi}(\xi)
$$

where

$$
\begin{gathered}
A_{c l 0}=\left[\begin{array}{ccc}
A_{0}+B_{0} D_{c} C & B_{0} C_{c} \\
B_{c} C & & A_{c}
\end{array}\right], \quad A_{c l, \xi}(\xi)=\left[\begin{array}{cc}
\tilde{A} \hat{\xi}_{n}+\tilde{B} \hat{\xi}_{m} D_{c} C & \tilde{B} \hat{\xi}_{m} C_{c} \\
\tilde{K} \hat{\xi}_{n} C & \tilde{L} \hat{\xi}_{r}
\end{array}\right]=\Phi_{1} \hat{\xi}_{p} \Phi_{2}, \quad p=2 n+m+r, \\
\Phi_{1}:=\left[\begin{array}{cccc}
\tilde{A} & \tilde{B} & 0 & 0 \\
0 & 0 & \tilde{L} & \tilde{K}
\end{array}\right], \quad \Phi_{2}=\Phi_{2,1}+\Phi_{2,2}, \quad \Phi_{2,1}:=\left[\begin{array}{cc}
I_{n} & 0 \\
D_{c} C & C_{c} \\
0 & 0 \\
C & 0
\end{array}\right], \quad \Phi_{2,2}:=\left[\begin{array}{ll}
0 & 0 \\
0 & 0 \\
0 & I_{n} \\
0 & 0
\end{array}\right] .
\end{gathered}
$$


We observe that the variable transformation (7) allows us to put in evidence the augmented state in the closed-loop dynamics $A_{c l}(\xi)$. This feature will be explored to get conditions independent of the states, under suitable constraints. As we will see later, the decomposition of $\Phi_{2}$ into $\Phi_{2,1}$ and $\Phi_{2,2}$ is convenient since each of these two matrices will be treated differently in order to develop convex design conditions.

A common approach in the literature of quadratic systems is to consider the representation of the closed-loop system (5) in a polytope ${ }^{28,24}$. In this case, the augmented state $\xi$ is characterized by the vertices of a polytope what leads the necessity to impose bounds on $x(t)$ and $x_{c}(t)$. Some works 28 justify this choice as part of the problem, that is, the polytope is determined by physical limitations and one wants to assure the asymptotically stability for all points belonging to this operational working space. Even in this framework, observe that imposing bounds on the states of the controller is rarely a given specification. If no operational work range is given, one needs to artificially impose a starting polytope and after that proceed with an iterative algorithm to systematically increase it, as much as possible, as proposed in 25 . This polytopic approach is not at the heart of our result but will be detailed in Section 4.3 to allow a comparison with our contribution. To be more precise, we adopt in this work the parameterization of the states as points inside an ellipsoid, which is a level set of a quadratic Lyapunov function, as proposed in 17 in the context of state feedback design for quadratic systems.

Let us finish this section with some lemmas which are instrumental for the further developments.

Lemma 1 (Finsler's Lemma ${ }^{29}$ ). Consider $w \in \mathbb{R}^{n}, M \in \mathbb{R}^{n \times n}, \mathcal{B} \in \mathbb{R}^{m \times n}$ with $\operatorname{rank}(\mathcal{B})<n$ and $\mathcal{B}_{\perp}$ a basis of the null space of $\mathcal{B}\left(B B_{\perp}=0\right)$. Then, the following statements are equivalent:
i. $w^{T} M w<0, \forall w \neq 0, \quad B w=0$.
ii. $\mathcal{B}_{\perp}^{T} M \mathcal{B}_{\perp}<0$.
iii. $\exists N \in \mathbb{R}^{n \times m}: \quad M+N \mathcal{B}+\mathcal{B}^{T} N^{T}<0$.

Lemma 2. Let a symmetric matrix $M_{0} \in \mathbb{R}^{n \times n}$ and matrices $M_{1} \in \mathbb{R}^{m \times n}$ and $M_{2} \in \mathbb{R}^{m \times n}$. Then the following conditions are equivalent:

$$
\begin{aligned}
& \text { i. } M_{0}+\operatorname{He}\left\{M_{1}^{T} M_{2}\right\}<0 . \\
& \text { ii. } \exists N \in \mathbb{R}^{n+m \times m}:\left[\begin{array}{cc}
M_{0} & M_{1}^{T} \\
M_{1} & 0
\end{array}\right]+\operatorname{He}\left\{N\left[M_{2}-I\right]\right\}<0 .
\end{aligned}
$$

Proof. The equivalence can be proved by applying Lemma 1 on the auxiliary matrices:

$$
M=\left[\begin{array}{cc}
M_{0} & M_{1}^{T} \\
M_{1} & 0
\end{array}\right], \quad \mathcal{B}_{\perp}=\left[\begin{array}{c}
I \\
M_{2}
\end{array}\right], \quad \mathcal{B}=\left[M_{2}-I\right] .
$$

\section{4 | MAIN RESULTS}

In this section, we present conditions to solve Problem 1 from the closed-loop dynamics described by (9).

Theorem 1. Consider a continuously differentiable function $V: \mathbb{R}^{2 n} \mapsto \mathbb{R}_{+}$given by $V(\xi)=\xi^{T} P \xi$. If there exist a positive definite matrix $P=P^{T} \in \mathbb{R}^{2 n \times 2 n}$ and matrices of the controller (3) such that the following conditions hold:

$$
\operatorname{He}\left\{P\left(A_{c l 0}+\Phi_{1}\left(I_{p} \otimes \xi\right) \Phi_{2}\right)\right\}<0 \quad \forall \xi \in S,
$$

where the set $S$ is defined by

$$
S:=\left\{\xi \in \mathbb{R}^{2 n}: V(\xi) \leq 1\right\}
$$

then:

- The origin is locally exponentially stable and $\mathcal{V}(\xi)=\xi^{T} P \xi$ is a (control-) Lyapunov function over $S$ for the system (5);

- $S \subset \mathbb{R}^{2 n}$ is a compact set which is positively invariant and exponentially contractive with respect to the augmented dynamics (5), i.e, for all $\xi(0) \in S, \lim _{t \rightarrow \infty} \xi(t)=0$; 
- The set $S_{0}=\left\{x \in \mathbb{R}^{n}:(x, 0) \in S\right\} \subset \mathbb{R}^{n}$, that can be rewritten as

$$
\mathcal{S}_{0}=\left\{x \in \mathbb{R}^{n}: x^{T}\left[\begin{array}{ll}
I_{n} & 0_{n}
\end{array}\right] P\left[\begin{array}{ll}
I_{n} & 0_{n}
\end{array}\right]^{T} x \leq 1\right\},
$$

is an estimate of the basin of attraction of the origin related to the system (5), when imposing a zero initial condition to the DOF controller (3), that is, $\forall \xi(0) \in S_{0} \times\{0\}, \lim _{t \rightarrow \infty} \xi(t)=0$.

Proof. Inequality (14) implies that there exists a sufficient small positive scalar $b$ such that the following inequality holds

$$
\dot{V}(\xi)=\frac{\partial V(\xi)}{\partial \xi} \dot{\xi}(t)=\frac{\partial V(\xi)}{\partial \xi} A_{c l}(\xi) \xi \leq-b \xi^{T} \xi, \quad \forall \xi \in S .
$$

Then the origin is exponentially stable with dynamics (5) starting from $S$. Since $S_{0} \times\{0\}$ is a subset of $S$, the trajectories of (5) converge exponentially to the origin for every initial condition $x(0) \in S_{0}$ and zero initial condition for the DOF controller. $S_{0}$ is an estimate of the basin of attraction of the origin.

Remark 1. It should be emphasized in particular that $S_{0}$ is not positively invariant. It is nevertheless interesting to notice that $S_{0}$ and $S$ are both obtained thanks to the same Lyapunov function $V$.

One observes that the inequality (14) is composed by two terms. The term $P A_{c l 0}$ appears in the classic dynamic output feedback problem and it can be linearized by a standard variable transformation ${ }^{30}$. The term $P \Phi_{1} \hat{\xi}_{p} \Phi_{2}$, representing $P A_{c l, \xi}(\xi)$, cannot be linearized with the same variable or congruence transformation and depends on the augmented state $\xi$. Since we want (14) to hold for all $\xi \in S$, the Petersen's Lemma ${ }^{31}$ can be used to eliminate the presence of the augmented state in the inequality. Although the application of the Schur complement yields an expression with an affine dependence on the decision variables, they are subjected to nonlinear constraints due to the presence of variables that usually disappears in the variable transformation ${ }^{30}$. The Finsler's Lemma, with a convenient choice for the slack variable, is finally used as a solution to obtain sufficient convex conditions to solve (14).

We are ready to present in the following subsections a solution to Problem 1 considering the nonlinear full-order DOF controller (3) (in Section 4.1) as well a linear one (in Section 4.2).

\section{1 | Quadratic output controller}

The following result presents a sufficient LMI condition to design (3) from Theorem 1

Theorem 2. If there exist symmetric positive definite matrices $Z \in \mathbb{R}^{n \times n}, Y \in \mathbb{R}^{n \times n}$, matrices $U \in \mathbb{R}^{n \times n}, Q \in \mathbb{R}^{n \times n}, F \in \mathbb{R}^{n \times r}$, $S \in \mathbb{R}^{m \times n}, R \in \mathbb{R}^{m \times r}, H_{i} \in \mathbb{R}^{n \times n}$ and $J_{i} \in \mathbb{R}^{n \times r}, i=1, \ldots, n$, and given scalars $\varepsilon>0$ and $\mu>0$ such that the following inequalities hold: $\tilde{P}=\left[\begin{array}{cc}Y & I \\ I & Z\end{array}\right]>0$ and

$$
\left[\begin{array}{cccc}
\operatorname{He}\{\Pi\} & \star & \star & \star \\
\tilde{\Phi}_{2} & -\varepsilon^{-1} I_{p} & \star & \star \\
\tilde{\Phi}_{1}^{T} & 0 & \mu I_{p} \otimes\left(\frac{\mu}{\varepsilon} \tilde{P}-\bar{P}-\bar{P}^{\prime}\right) & \star \\
\tilde{P} & \Phi_{2,2}^{T} & 0 & -\operatorname{He}\{\bar{P}\}
\end{array}\right]<0,
$$

where

$$
\begin{aligned}
& \Pi=\left[\begin{array}{cc}
A_{0} Y+B_{0} S & A_{0}+B_{0} R C \\
Q & Z A_{0}+F C
\end{array}\right], \quad \tilde{\Phi}_{2}=\left[\begin{array}{cc}
Y & I_{n} \\
S & R C \\
0 & 0 \\
C Y & C
\end{array}\right],
\end{aligned}
$$

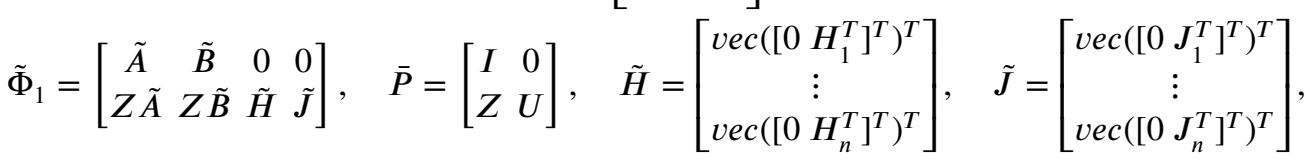


and $\Phi_{2,2}$ defined in (11). Then, Inequality (14) is ensured with the controller (3) defined by

$$
\begin{gathered}
{\left[\begin{array}{cc}
A_{c} & B_{c} \\
C_{c} & D_{c}
\end{array}\right]=\left[\begin{array}{cc}
U & Z B_{0} \\
0 & I
\end{array}\right]^{-1}\left[\begin{array}{cc}
Q-Z A_{0} Y & F \\
S & R
\end{array}\right]\left[\begin{array}{cc}
V^{T} & 0 \\
C Y & I
\end{array}\right]^{-1}, \quad V=(I-Y Z) U^{-T},} \\
\tilde{L}=U^{-1} \tilde{H}, \quad \tilde{K}=U^{-1} \tilde{J}
\end{gathered}
$$

and Lyapunov matrix $P$ given by

$$
P=\left[\begin{array}{cc}
Z & U \\
U^{T} & -U^{T} Y V^{-T}
\end{array}\right]
$$

Before presenting the complete proof of Theorem 2, we would like to consider one of the main difficulty of inequality (14): that is, the dependence on $\xi$ of the second term of (14). Lemma 3 provides then a suitable upper bound of this second term, independent of $\xi$.

Lemma 3. For a given positive definite matrix $P=P^{T} \in \mathbb{R}^{2 n \times 2 n}$, for all $\xi \in S=\left\{\xi \in \mathbb{R}^{2 n}: \xi^{T} P \xi \leq 1\right\}$ and for any positive scalar $\varepsilon$, the following upper bound, independent of $\xi$, holds:

$$
\operatorname{He}\left\{P \Phi_{1}\left(I_{p} \otimes \xi\right) \Phi_{2}\right\} \leq \frac{1}{\varepsilon} P \Phi_{1}\left(I_{p} \otimes P^{-1}\right) \Phi_{1}^{T} P+\varepsilon \Phi_{2}^{T} \Phi_{2} .
$$

Proof. Following 17 , Lemma 1 , every point on the interior of an ellipsoid $S=\left\{\xi \in \mathbb{R}^{2 n}: V(\xi) \leq 1\right\}$ can be parameterized by $\xi=P^{-1 / 2} \Delta v$, with $\Delta^{T} \Delta \leq I$ and $v$ a vector such that $v^{T} v=1$. Using Petersen's Lemma 31 and the parameterization $\xi=P^{-1 / 2} \Delta v, \forall \xi \in S$, one can write

$$
\begin{aligned}
\operatorname{He}\left\{P \Phi_{1} \hat{\xi}_{p} \Phi_{2}\right\} & =\operatorname{He}\left\{P \Phi_{1}\left(I_{p} \otimes P^{-1 / 2}\right)\left(I_{p} \otimes \Delta v\right) \Phi_{2}\right\} \\
& \leq \frac{1}{\varepsilon} P \Phi_{1}\left(I_{p} \otimes P^{-1 / 2}\right)\left(I_{p} \otimes P^{-1 / 2}\right) \Phi_{1}^{T} P+\varepsilon \Phi_{2}^{T}\left(I_{p} \otimes v^{T} \Delta^{T}\right)\left(I_{p} \otimes \Delta v\right) \Phi_{2} \\
& \leq \frac{1}{\varepsilon} P \Phi_{1}\left(I_{p} \otimes P^{-1}\right) \Phi_{1}^{T} P+\varepsilon \Phi_{2}^{T} \Phi_{2},
\end{aligned}
$$

where $\varepsilon>0$.

Proof of Theorem 2 In order to ensure the condition (14) of Theorem 11, thanks to Lemma 3, we would like to consider a sufficient condition, which is independent of $\xi \in S$, that is

$$
\operatorname{He}\left\{P A_{c l 0}\right\}+\frac{1}{\varepsilon} P \Phi_{1}\left(I_{p} \otimes P^{-1}\right) \Phi_{1}^{T} P+\varepsilon \Phi_{2}^{T} \Phi_{2}<0 .
$$

The latter inequality is strongly nonlinear in the variables (not only quadratic or bilinear), and applying straightforwardly the standard linearization techniques fail to linearize it. Applying first a Schur complement will allow to separate the nonlinear terms to combine adequately distinct linearization techniques, that results in:

$$
\left[\begin{array}{ccc}
\operatorname{He}\left\{P A_{c l 0}\right\} & \Phi_{2}^{T} & P \Phi_{1} \\
\star & -\varepsilon^{-1} I_{p} & 0 \\
\star & \star & -\varepsilon I_{p} \otimes P
\end{array}\right]<0 .
$$

We propose a linearization in two consecutive steps.

\section{Step 1}

This step will be focused on linearizing the bilinear terms $P A_{c l 0}$ and $P \Phi_{1}$. For this purpose, we follow the methodology provided in ${ }^{30}$. First, let us introduce the partition of the Lyapunov matrix $P$ :

$$
P=\left[\begin{array}{cc}
Z & U \\
U^{T} & \mathbf{\square}
\end{array}\right], \quad P^{-1}=\left[\begin{array}{cc}
Y & V \\
V^{T} & \mathbf{\square}
\end{array}\right],
$$

associate with the constraint $Y Z+V U^{T}=I$, and the change of variables

$$
\left[\begin{array}{ll}
Q & F \\
S & R
\end{array}\right]:=\left[\begin{array}{cc}
U & Z B_{0} \\
0 & I
\end{array}\right]\left[\begin{array}{ll}
A_{c} & B_{c} \\
C_{c} & D_{c}
\end{array}\right]\left[\begin{array}{cc}
V^{T} & 0 \\
C Y & I
\end{array}\right]+\left[\begin{array}{cc}
Z A_{0} Y & 0 \\
0 & 0
\end{array}\right] .
$$

Thanks to this decomposition, we can express the desired sets $S$ and $S_{0}$ as the following ellipsoids: $S=\left\{\xi \in \mathbb{R}^{2 n}: \xi^{T} P \xi \leq 1\right\}$ and $S_{0}=\left\{x \in \mathbb{R}^{n}: x^{T} Z x \leq 1\right\}$. 

by

By considering that matrix $V$ is invertible, that will be imposed in the following, we define the non singular matrix $T$ defined

$$
T:=\left[\begin{array}{cc}
Y & I \\
V^{T} & 0
\end{array}\right]
$$

The congruence transformation is adopted to the size of inequality (22) by $\Lambda_{1}:=\operatorname{diag}\left(T, I_{p}, I_{2 n p}\right)$ and its transpose, respectively, yielding

$$
\left[\begin{array}{ccc}
\operatorname{He}\{\Pi\} & \tilde{\Phi}_{2}^{T}+T^{T} \Phi_{2,2}^{T} & \tilde{\Phi}_{1} \\
\star & -\varepsilon^{-1} I_{p} & 0 \\
\star & \star & -\varepsilon I_{p} \otimes P
\end{array}\right]<0
$$

with the following notations $\Pi:=T^{T} P A_{c l 0} T, \tilde{\Phi}_{2}:=\Phi_{2,1} T, \tilde{\Phi}_{1}:=T^{T} P \Phi_{1}, \tilde{H}:=U \tilde{L}$ and $\tilde{J}:=U \tilde{K}$, expressed as (18).

One observes in (26) that the terms $P, \Pi, \tilde{\Phi}_{2}$ and $\tilde{\Phi}_{1}$ have affine dependence on the variables $Y, Z, S, F, Q, R, U, \tilde{H}$ and $\tilde{J}$, where $\tilde{H}$ and $\tilde{J}$ are matrices that linearize the bilinear products $U \tilde{L}$ and $U \tilde{K}$. However, the term $\Phi_{2,2} T$ still depends on matrix $V$ that is related to the nonlinear restriction $Y Z+V U^{T}=I$. The second step will be dedicated to linearize this term and avoid dependence on matrix $V$.

\section{Step 2}

This step is based on a rewriting of inequality (26) in the form of (12) in Lemma2 that allows to modify the terms bilinear in the variables into a product between a variable and a slack variable. Finally, a suitable choice of the slack variables provides sufficient conditions ensuring (26) as LMI avoiding the variable $V$. In details, (26) is equivalent to inequality (12) with

$$
M_{0}=\left[\begin{array}{ccc}
\operatorname{He}\{\Pi\} & \tilde{\Phi}_{2}^{T} & \tilde{\Phi}_{1} \\
\star & -\varepsilon^{-1} I_{p} & 0 \\
\star & \star & 0
\end{array}\right], M_{1}=\left[\begin{array}{ccc}
0 & \Phi_{2,2}^{T} & 0 \\
0 & 0 & -\frac{\varepsilon}{2} I_{p} \otimes P
\end{array}\right], M_{2}=\left[\begin{array}{ccc}
T & 0 & 0 \\
0 & 0 & I_{2 n p}
\end{array}\right] .
$$

The slack variable in Lemma 2 is $N \in \mathbb{R}^{4 n(1+p)+p \times 2 n(1+p)}$. Let us denote $\Lambda:=\operatorname{diag}\left(I_{2 n}, I_{p}, I_{2 n p}, \Lambda_{2}\right)$ with $\Lambda_{2}:=\operatorname{diag}\left(I_{2 n}, I_{p} \otimes T\right)$. By pre- and post-multiplying (13) by $\Lambda^{T}$ and its transpose, respectively, yields

$$
\left[\begin{array}{cc}
M_{0} & \tilde{M}_{1}^{T} \\
\tilde{M}_{1} & 0
\end{array}\right]+\operatorname{He}\left\{\Lambda^{T} N\left[M_{2}-\Lambda_{2}\right]\right\}<0
$$

with

$$
\tilde{M}_{1}=\left[\begin{array}{ccc}
0 & \Phi_{2,2}^{T} & 0 \\
0 & 0 & -\frac{\varepsilon}{2} I_{p} \otimes \bar{P}
\end{array}\right], \quad \bar{P}:=T^{T} P .
$$

The equivalence is now broken by selecting a convenient choice of the slack variable $N$ in order to cancel the dependence on $V$. Therefore, the following possible structure of $N$ is proposed (this is not the unique option of course):

$$
N=\left[\begin{array}{c}
0_{2 n+p \times 2 n(1+p)} \\
\frac{0-\mu I_{p} \otimes T^{T}}{I(\varepsilon)}
\end{array}\right]\left[\begin{array}{cc}
T^{T} P & 0 \\
0 & I_{p} \otimes P
\end{array}\right], \quad I(\varepsilon):=\left[\begin{array}{cc}
I_{2 n} & 0 \\
0 & \frac{\varepsilon}{2} I_{2 n p}
\end{array}\right],
$$

where $\mu$ is a positive scalar representing an extra degree of freedom. Then, one has

$$
\begin{aligned}
\Lambda^{T} N\left[M_{2}-\Lambda_{2}\right] & =\left[\frac{0}{\frac{0-\mu I_{p} \otimes T^{T}}{\Lambda_{2}^{T} I(\varepsilon)}}\right]\left[\begin{array}{cc}
T^{T} P & 0 \\
0 & I_{p} \otimes P
\end{array}\right]\left[M_{2} \mid-\Lambda_{2}\right] \\
& =\left[\frac{0}{0-\mu I}\right]\left[\begin{array}{ccc|cc}
\tilde{P} & 0 & 0 & -\bar{P} & 0 \\
0 & 0 & I_{p} \otimes \bar{P} & 0 & -I_{p} \otimes \tilde{P}
\end{array}\right],
\end{aligned}
$$

where $\tilde{P}:=T^{T} P T=\left[\begin{array}{ll}Y & I \\ I & Z\end{array}\right]>0$ 
Replacing the expression (30) in 28 yields to

$$
\left[\begin{array}{cccc|c}
\operatorname{He}\{\Pi\} & \star & \star & \star & \star \\
\tilde{\Phi}_{2} & -\varepsilon^{-1} I_{p} & \star & \star & \star \\
\tilde{\Phi}_{1}^{T} & 0 & -\mu \operatorname{He}\left\{I_{p} \otimes \bar{P}\right\} & \star & \star \\
\tilde{P} & \Phi_{2,2}^{T} & 0 & -\operatorname{He}\{\bar{P}\} & \star \\
\hline 0 & 0 & \mu I_{p} \otimes \tilde{P} & 0 & -\varepsilon I_{p} \otimes \tilde{P}
\end{array}\right]<0,
$$

and, finally, by applying a Schur complement on the last block line and column, to the inequality (17).

To end the proof, the invertibility of $U$, and therefore of $V$, are guaranteed by $U+U^{T}>0$ in block (4,4) of $(17)$ and thus the gains (19)-20) are well defined.

Remark 2. It is noteworhty that sufficient conditions in Theorem 2 are closely related to the choice of the structure of the slack variable $N$ in (29). The level of performance, and in particular the size of the estimate of the basin of attraction in closed form, is affected by this selection and may be improved. It is also of interest to underline that the matrix $N$ depends on matrix $V$ to avoid the global dependence on matrix $V$ of the inequality (28).

\section{2 | Linear output controller}

This section is devoted to the particular framework of linear dynamic output feedback of the form:

$$
\begin{aligned}
\dot{x}_{c}(t) & =A_{c} x_{c}(t)+B_{c} y(t), \\
u(t) & =C_{c} x_{c}(t)+D_{c} y(t) .
\end{aligned}
$$

Instead of applying straightforwardly the results of the previous subsection to the linear case, the considered viewpoint here is to follow the same methodology in addition of judicious modifications or specificities in order to highlight the simplified conditions. The first structural simplification is emphasized by the fact that the dynamic of the extended state contains a matrix depending only on the state $x$ and not on the extended state $\xi$. To be more precise, the dependence on the state of the controller is adequately circumvented by rearranging the terms as detailed in 25 :

$$
\dot{\xi}(t)=A_{c l, \ell}(x) \xi(t),
$$

where

$$
A_{c l, \ell}(x)=A_{c l 0}+\Theta_{1}\left(I_{p_{\ell}} \otimes x\right) \Theta_{2}
$$

with $A_{c l 0}$ defined by $(10), p_{\ell}=n+m$,

$$
\Theta_{1}:=\left[\begin{array}{cc}
\tilde{A}_{\ell} & \tilde{B}_{\ell} \\
0 & 0
\end{array}\right], \quad \Theta_{2}:=\left[\begin{array}{cc}
I_{n} & 0 \\
D_{c} C & C_{c}
\end{array}\right]
$$

and

$$
\tilde{A}_{\ell}:=\left[\begin{array}{lll}
\operatorname{vec}\left(A_{1}\right) & \cdots & \operatorname{vec}\left(A_{n}\right)
\end{array}\right]^{T} \in \mathbb{R}^{n \times n^{2}}, \quad \tilde{B}_{\ell}:=\left[\begin{array}{lll}
\operatorname{vec}\left(B_{1}\right) & \cdots & \operatorname{vec}\left(B_{n}\right)
\end{array}\right]^{T} \in \mathbb{R}^{n \times n m} .
$$

Following similar steps presented in Section 4.1 next result presents sufficient conditions to the design of (31) and the determination of a region $S_{0}$ such that the trajectories of closed-loop system starting from any initial condition $\left(x(0), x_{c}(0)\right) \in$ $S_{0} \times\{0\}$ converge exponentially towards the origin.

Corollary 1. If there exist symmetric positive definite matrices $Z \in \mathbb{R}^{n \times n}, Y \in \mathbb{R}^{n \times n}$, matrices $Q \in \mathbb{R}^{n \times n}, F \in \mathbb{R}^{n \times r}$, $S \in \mathbb{R}^{m \times n}, R \in \mathbb{R}^{m \times r}$, and given scalars $\varepsilon>0$ and $\mu>0$, such that the following inequalities hold: $\tilde{P}=\left[\begin{array}{ll}Y & I \\ I & Z\end{array}\right]>0$ and

$$
\left[\begin{array}{ccc}
\operatorname{He}\{\Pi\} & \star & \star \\
\tilde{\Theta}_{2} & -\varepsilon^{-1} I_{p_{\ell}} & \star \\
\tilde{\Theta}_{1}^{T} & 0 & \mu I_{p_{\ell}} \otimes\left(\frac{\mu}{\varepsilon} Y-2 I\right)
\end{array}\right]<0
$$

where

$$
\tilde{\Theta}_{1}=\left[\begin{array}{cc}
\tilde{A}_{\ell} & \tilde{B}_{\ell} \\
Z \tilde{A}_{\ell} & Z \tilde{B}_{\ell}
\end{array}\right], \quad \tilde{\Theta}_{2}=\left[\begin{array}{cc}
Y & I_{n} \\
S & R C
\end{array}\right],
$$

and $\Pi$ given by (18a). Then, the origin of the closed-loop system (32) is locally exponentially stable with controller (31) with gains given by (19) with $U$ and $V$ such that $Y Z+V U^{T}=I$. Moreover, for any initial condition $\left(x(0), x_{c}(0)\right) \in S_{0} \times\{0\}$, with $S_{0}$ as in (16), the resulting trajectories of system (5) exponentially converge towards the origin. 
Proof. First, observe that, considering the linear controller (31), condition (14) becomes

$$
\operatorname{He}\left\{P\left(A_{c l 0}+\Theta_{1}\left(I_{p_{\ell}} \otimes x\right) \Theta_{2}\right\}<0, \quad \forall \xi \in S,\right.
$$

with $\Theta_{1}$ and $\Theta_{2}$ given by (34). From Lemmaß], if $\xi \in S$, then $x=\left[\begin{array}{ll}I & 0\end{array}\right] P^{-1 / 2} \Delta v$ and a sufficient condition for (38) is given by

$$
\mathrm{He}\left\{P A_{c l 0}\right\}+\frac{1}{\varepsilon} P \Theta_{1}\left(I_{p_{\ell}} \otimes Y\right) \Theta_{1}^{T} P+\varepsilon \Theta_{2}^{T} \Theta_{2}<0 .
$$

By applying the Schur complement, one gets

$$
\left[\begin{array}{ccc}
\operatorname{He}\left\{P A_{c l 0}\right\} & \Theta_{2}^{T} & P \Theta_{1} \\
\star & -\varepsilon^{-1} I_{p_{\ell}} & 0 \\
\star & \star & -\varepsilon I_{p_{\ell}} \otimes Y^{-1}
\end{array}\right]<0
$$

As before, pre- and post-multiplying (39) by $\Lambda_{1}$ and its transpose, respectively, and rewritten it as (12) with

$$
M_{0}=\left[\begin{array}{ccc}
\operatorname{He}\{\Pi\} & \star & \star \\
\tilde{\Theta}_{2} & -\varepsilon^{-1} I_{p_{\ell}} \star \\
\tilde{\Theta}_{1}^{T} & 0 & 0
\end{array}\right], \quad M_{1}=\left[\begin{array}{lll}
0 & 0 & -\frac{\varepsilon}{2} I_{p_{\ell}} \otimes Y^{-1}
\end{array}\right], \quad M_{2}=\left[\begin{array}{lll}
0 & 0 & I_{n p_{\ell}}
\end{array}\right],
$$

with $\tilde{\Theta}_{2}:=\Theta_{2} T, \tilde{\Theta}_{1}:=T^{T} P \Theta_{1}, T$ given by $\left[25\right.$, then, by Lemma2, there exists a matrix $N \in \mathbb{R}^{2 n\left(1+p_{\ell}\right)+p_{\ell} \times n p_{\ell}}$ such that (39) is equivalent to (13).

Let $\Lambda=\operatorname{diag}\left(I_{2 n}, I_{p_{\ell}}, I_{n p_{\ell}}, I_{p_{\ell}} \otimes Y\right)$, then pre- and post-multiplying (13) by $\Lambda^{T}$ and its transpose, respectively, one has

$$
\left[\begin{array}{cc}
M_{0} & \tilde{M}_{1}^{T} \\
\tilde{M}_{1} & 0
\end{array}\right]+\operatorname{He}\left\{\Lambda^{T} N\left[\begin{array}{lll}
0 & 0 & I_{n p_{\ell}} \mid-I_{p_{\ell}} \otimes Y
\end{array}\right]\right\}<0,
$$

with

$$
\tilde{M}_{1}=\left[\begin{array}{lll}
0 & 0 & -\frac{\varepsilon}{2} \\
I_{n p_{\ell}}
\end{array}\right] .
$$

The following convenient choice for the slack variable $N$ is proposed:

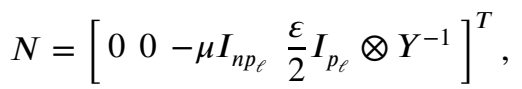

where the positive scalar parameter $\mu$ is introduced as an extra degree of freedom.

Condition (41) with $N$ given by (42) is equivalent to

$$
\left[\begin{array}{cccc}
\operatorname{He}\{\Pi\} & \star & \star & \star \\
\tilde{\Theta}_{2} & -\varepsilon^{-1} I_{p_{\ell}} & \star & \star \\
\tilde{\Theta}_{1}^{T} & 0 & -2 \mu I_{n p_{\ell}} & \star \\
0 & 0 & \mu I_{p_{\ell}} \otimes Y & -\varepsilon I_{p_{\ell}} \otimes Y
\end{array}\right]<0 .
$$

Applying the Schur complement in the above inequality, one obtains (36).

Remark 3. We would like to observe that (36) is not roughly speaking an extraction of inequality (17), that is, there is no singular congruence transformation to obtain (36) from (17). That can be explained by the fact that the closed form $A_{c l}(\xi)$ depends on the extended state $\xi$ and that $A_{c l, \ell}(x)$ only on the state of the system $x$.

A polytopic approach, used in 28,25 , in the context of bilinear systems, can also be considered to provide a solution to Problem 1 We detail in the sequence the design of the linear controller (31) following this approach allowing a comparison with our main contribution. For the sake of brevity, the design of the quadratic controller (3) following the polytopic approach is not detailed.

\section{3 | Polytopic approach}

The main feature of the polytopic approach consists in defining a priori a polyhedral set and considering the initial conditions belonging to an invariant region contained in this set.

Consider a polyhedral set $\chi_{0} \subset \mathbb{R}^{n}$ given by

$$
\chi_{0}=\left\{x \in \mathbb{R}^{n}: \Omega x \preceq \mathbf{1}\right\},
$$

where $\Omega \in \mathbb{R}^{g \times n}, n \leq g, \operatorname{rank}\left(Q_{0}\right)=n$ and $\mathbf{1} \in \mathbb{R}^{g}$. Observe that $0 \in \chi_{0}$. The set $\chi_{0}$ can also be defined in terms of its $\kappa$ vertices,

$$
\chi_{0}=\operatorname{co}\left\{v_{1}, v_{2}, \ldots, v_{\kappa}\right\} \text {, }
$$


where $v_{j} \in \mathbb{R}^{n}, j=1, \ldots, \kappa$, can be obtained from $\Omega$. Note that in the case where $\chi_{0}$ is a symmetric hyper-rectangle, we have $g=2 n$ and $\kappa=2^{n}$.

Hence, the polyhedral set $\chi_{0}$ induces the following $n \times n$-matrix valued polytope

$$
\mathcal{P}=\operatorname{co}\left\{A_{c l, \ell}\left(v_{j}\right), j=1, \ldots, \kappa\right\},
$$

such that $x \in \chi_{0}$ implies $A_{c l, \ell}(x) \in \mathcal{P}$.

Since there is no restriction for the state $x_{c}$ to induce $\mathcal{P}$, the domain $\chi_{0}$ yields the polyhedral set $\chi=\chi_{0} \times \mathbb{R}^{n}$. Following Theorem 2 of $\frac{25}{5}$, the origin of (32) is locally exponentially stable if there exist a continuously differentiable function $V: \mathbb{R}^{2 n} \mapsto$ $\mathbb{R}_{+}$, given by $V(\xi)=\xi^{T} P \xi, P=P^{T}>0 \in \mathbb{R}^{2 n \times 2 n}$, and a positive scalar $b$ such that the following conditions hold:

$$
\begin{gathered}
\frac{\partial V(\xi)}{\partial \xi} A_{c l, \ell}\left(v_{j}\right) \xi<-b \xi^{T} \xi, \quad \forall \xi \in \chi, j=1, \ldots, \kappa, \\
S \subseteq \chi,
\end{gathered}
$$

where the set $S$, defined in (15), is a compact set which is positively invariant and exponentially contractive with respect to the augmented dynamics (32), i.e, for all $\xi(0) \in S, \lim _{t \rightarrow \infty} \xi(t)=0$. The following result presents a sufficient LMI condition to design (31) such that (44) holds.

Theorem 3. If there exist symmetric positive definite matrices $Z \in \mathbb{R}^{n \times n}, Y \in \mathbb{R}^{n \times n}$, matrices $Q \in \mathbb{R}^{n \times n}, F \in \mathbb{R}^{n \times r}, S \in \mathbb{R}^{m \times n}$, $R \in \mathbb{R}^{m \times r}$, and a given scalar $\mu>0$, such that the following inequalities hold: $\tilde{P}=\left[\begin{array}{ll}Y & I \\ I & Z\end{array}\right]>0$ and

$$
\begin{gathered}
{\left[\begin{array}{cc}
\operatorname{He}\{\Pi\} & \tilde{\Theta}_{2}^{T}+\mu \tilde{\Theta}_{1}\left(I_{p_{\ell}} \otimes v_{i}\right) \\
\star & -2 \mu I_{p_{\ell}}
\end{array}\right]<0, \quad i=1, \ldots, \kappa,} \\
{\left[\begin{array}{ccc}
1 & \Omega_{(i)}\left[\begin{array}{ll}
Y & I
\end{array}\right] \\
\star & \tilde{P}
\end{array}\right] \geq 0, \quad i=1, \ldots, g,}
\end{gathered}
$$

where $\tilde{\Theta}_{1}$ and $\tilde{\Theta}_{2}$ are given by (37) and $\Pi$ by (18a). Then, the origin of the closed-loop system (32) is locally exponentially stable with controller (31) with gains given by (19) with $U$ and $V$ such that $Y Z+V U^{T}=I$. Moreover, for any initial condition $\left(x(0), x_{c}(0)\right) \in S_{0} \times\{0\}$, with $S_{0}$ as in (16), the resulting trajectories of system (32) exponentially converge towards the origin.

Proof. Observe that the inequality

$$
\operatorname{He}\left\{P\left(A_{c l 0}+\Theta_{1}\left(I_{p_{\ell}} \otimes v_{j}\right) \Theta_{2}\right\}<0, \quad j=1, \ldots, \kappa,\right.
$$

with $\Theta_{1}$ and $\Theta_{2}$ given by (34), implies (44a). Pre- and post-multiplying the above inequality by $T^{T}$, given in (25), and its transpose, respectively, one has

$$
\operatorname{He}\left\{\Pi+\tilde{\Theta}_{1}\left(I_{p_{\ell}} \otimes v_{j}\right) \tilde{\Theta}_{2}\right\}<0,
$$

that can be rewritten as (12) with $M_{0}=\operatorname{He}\{\Pi\}, M_{1}=\tilde{\Theta}_{2}$ and $M_{2}^{T}=\tilde{\Theta}_{1}\left(I_{p_{\ell}} \otimes v_{j}\right)$. Then, by Lemma 2 , there exists a matrix $N \in \mathbb{R}^{2 n+p_{\ell} \times n p_{\ell}}$ such that (13) is equivalent to (47). With the following convenient choice for the slack variable $N$

$$
N=\left[\begin{array}{c}
0 \\
\mu I
\end{array}\right]
$$

where $\mu$ is a positive scalar parameter, (13) recovers (45).

Condition

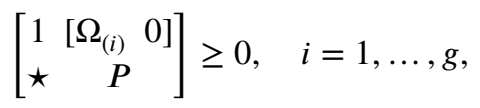

yields $[44 \mathrm{~b})^{32}$ with the set $\chi$ constructed from $\chi_{0}$ as $\chi=\left\{\xi \in \mathbb{R}^{2 n}:\left[\begin{array}{ll}\Omega & 0_{g \times n}\end{array}\right] \xi \leq \mathbf{1}\right\}$. Pre- and post-multiplying the above inequality by $\operatorname{diag}\left(I, T^{T}\right)$ and its transpose, respectively, one obtains (46).

Remark 4. As pointed out in Remark 2 , the choice of $N$ is not unique. More intricate structures could be proposed yielding extra parameters for a line search. However, we have verified in numerical experiments that (48) yields a good trade-off between feasibility and computational burden.

Remark 5. The choice of the set $\chi_{0}$ is far from trivial and the complexity increases with the order of the system. To circumvent arbitrary choices for $\chi_{0}$, many authors $\frac{16,24,25}{2}$ proposed iterative procedures to progressively increase the size of the polytope. These algorithms do not have any guaranty of convergence, involve many variables and are sensitive to the initial guess of $\chi_{0}$. 
It is out of the scope of this paper to describe or propose iterative algorithms to increase $\chi_{0}$ and the reader may refer to the aforementioned works. Finally, it is worthy to highlight the advantage of the methods proposed in Sections 4.1 and 4.2 that do not require the guess of $\chi_{0}$ and can optimize the set $S_{0}$ without the need of iterative procedures.

\section{I COMPUTATIONAL AND NUMERICAL ISSUES}

In this section, we present numerical experiments to illustrate the proposed results. The first example presents the estimation of the region of attraction using Theorems 2 and 3 The aim is to compare the size of $S_{0}$ for techniques that represent states inside an ellipsoid (Theorem 2) or a polytope (Theorem 3). The second example shows the trajectories of the closed-loop system for initial conditions in the boundary of $S_{0}$ with the controller designed by Corollary 1 to highlight the aspect that $S_{0}$ is not an invariant set. Before performing the numerical experiments, we present some remarks concerning the maximization of the estimate of the domain of attraction of the origin and the numerical implementation of Theorem 2 , Corollary 1 and Theorem 3.

Remark 6. A way to indirectly maximize the set $S_{0}$ defined in (16) is to minimize the trace of $Z^{22}$. For given positive scalars $\varepsilon$ and $\mu$, the maximization of the estimate of the basin of attraction $S_{0}$ is obtained by solving the following optimization problem:

$$
\min \operatorname{Trace}(\mathrm{Z})
$$

subjected to (17), (36) or (45)-(46). We have observed that the maximization of $S_{0}$ by (49) may lead to high gains for controllers (3) and (31) that can be undesirable from a practical point of view. To balance the trade off between the size of $S_{0}$ and the magnitude of the gains, an lower bound for the trace of $Z$ can be add as constraint to the LMI problem.

Remark 7. Theorem 2, Corollary 1 and Theorem 3 are LMIs for fixed values of the scalar parameters $\varepsilon$ and $\mu$. The choice of $\varepsilon$ and $\mu$ plays an important role in the feasibility and the estimation of $S_{0}$. A search in a given set of values can be performed and the conservativeness of the obtained estimate of the basin of attraction can be compared numerically in terms of the trace of $Z$. For the sake of conciseness, we restrict the search of the scalars parameters in the LMI conditions to the set $\{0.1,1,10\}$ yielding satisfactory results in terms of feasibility and estimation of the region of attraction $S_{0}$.

Example 1. Consider the quadratic system (1) with matrices:

$$
\begin{gathered}
{\left[A_{0}\left|A_{1}\right| A_{2}\right]=\left[\begin{array}{cc|cc|cc}
1 / 6 & 1 & 1 & 0.4 & 0.8 & 1 \\
0 & 1 / 6 & 0.4 & 0.2 & 1 & 0.8
\end{array}\right],} \\
{\left[B_{0}\left|B_{1}\right| B_{2}\right]=\left[\begin{array}{cc|cc|cc|c}
-4 & 2 & 1 & 0.8 & 0.4 & 1 \\
-2 & -2 & 0.4 & 1 & 0.2 & 0.8
\end{array}\right], \quad C=\left[\begin{array}{cc}
1 & 0 \\
0.2 & 0.9
\end{array}\right],}
\end{gathered}
$$

where the matrices $A_{0}, B_{0}, B_{1}$ and $B_{2}$ are taken from the bilinear system presented in 16 .

We would like to design the DOF controller (3) that locally exponentially stabilizes system (1) and to determine an estimate of the basin of attraction $S_{0} \subseteq \mathbb{R}^{2}$.

The following gains of the quadratic DOF controller (3) are obtained by Theorem 2 with $\varepsilon=\mu=10$ :

$$
\begin{gathered}
A_{c}=\left[\begin{array}{cc}
-303.7 & -281.6 \\
-498 & -968.5
\end{array}\right], B_{c}=\left[\begin{array}{cc}
-2.837 & 0.07329 \\
0.5376 & -3.263
\end{array}\right], \\
C_{c}=\left[\begin{array}{ll}
-429.9 & -670.3 \\
-177.4 & -419.9
\end{array}\right], D_{c}=\left[\begin{array}{cc}
-0.06004 & 0.06066 \\
0.05391 & -0.06758
\end{array}\right], \\
L_{1}=\left[\begin{array}{cc}
0.229 & 9.936 \cdot 10^{-6} \\
0.2978 & -0.2802
\end{array}\right], K_{1}=\left[\begin{array}{ll}
-0.2182 & -0.3799 \\
0.07298 & -0.3382
\end{array}\right], \\
L_{2}=\left[\begin{array}{cc}
-0.01748 & -0.1062 \\
0.1799 & -0.03577
\end{array}\right], K_{2}=\left[\begin{array}{ll}
0.8418 & -0.3428 \\
0.3044 & -0.2303
\end{array}\right]
\end{gathered}
$$

and

$$
Z=\left[\begin{array}{cc}
4.547 & 0.0667 \\
0.0667 & 7.714
\end{array}\right]
$$

To compare the conditions for the design of controller (3) using Theorem 2 and the polytopic approach described in Subsection 4.3, we present in Figure 1 the estimate of the domain of attraction of the origin for the closed-loop system obtained with both methods. To obtain the largest $S_{0}$, we employ the optimization problem described in Remark 6 , a search on the 
scalar values in the set $\{0.1,1,10\}$, and the use of the largest polyhedral region $\chi_{0}$ such that the conditions of Theorem 3 are feasible. We obtained the largest estimation of $S_{0}$ with $\varepsilon=\mu=0.1$ in Theorem 2 and, in Theorem 3 , with $\mu=1$ and $\chi_{0}=\left\{x \in \mathbb{R}^{2}:\left|x_{i}\right| \leq 1.01, i=1,2\right\}$. We can verify in Figure 1 that the parameterization of the states as points belonging to the interior of an ellipsoid provided a better estimation for $S_{0}$ without the drawback of fixing a polytope or implementing iterative algorithms to enlarge it.

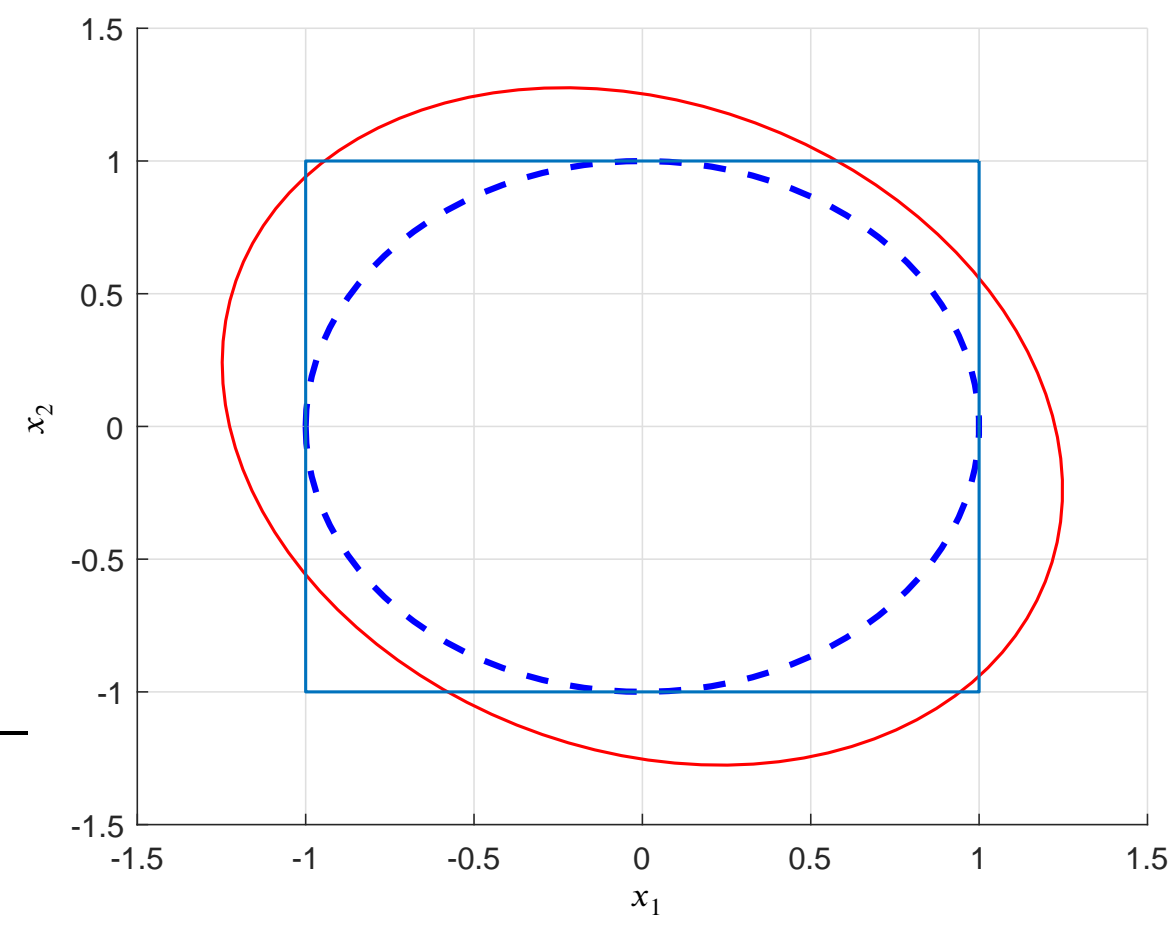

FIGURE 1 Estimation of the region of attraction $S_{0}$ for Example 1 with controllers designed by Theorem 2 with $\varepsilon=\mu=0.1$ (solid red line) and the conditions of Theorem 3 with $\mu=1$ and $\chi_{0}=\left\{x \in \mathbb{R}^{2}:\left|x_{i}\right| \leq 1.01, i=1,2\right\}$ (dashed blue line). In both methods, we considered the optimization problem described in Remark 6

Example 2. Consider the following quadratic system given in 24,17 :

$$
\dot{x}(t)=\left[\begin{array}{cc}
0 & 1+0.2 x_{2}(t) \\
2 x_{1}(t)+x_{2}(t) & x_{1}(t)
\end{array}\right] x(t)+\left[\begin{array}{l}
0 \\
1
\end{array}\right] u(t),
$$

$x(t)=\left(x_{1}(t), x_{2}(t)\right)$, yielding the matrices

$$
\left[A_{0}\left|A_{1}\right| A_{2}\right]=\left[\begin{array}{ll|lc|cc}
0 & 1 & 0 & 0 & 2 & 1 \\
0 & 0 & 0 & 0.2 & 1 & 0
\end{array}\right], \quad\left[B_{0}\left|B_{1}\right| B_{2}\right]=\left[\begin{array}{l|l|l}
0 & 0 & 0 \\
1 & 0 & 0
\end{array}\right] .
$$

We have adapted the state feedback control problem from ${ }^{24,17}$ to the output feedback one by considering the measurement of the state $x_{1}$ only, that is, $C=\left[\begin{array}{ll}1 & 0\end{array}\right]$.

The design of the linear DOF controller (31) by Corollary 1 with $\varepsilon=1$ and $\mu=10$ provides the following gains:

$$
\begin{gathered}
A_{c}=\left[\begin{array}{cc}
318.4 & 4254 \\
-58.78 & -773.8
\end{array}\right], B_{c}=\left[\begin{array}{l}
-789.7 \\
-233.9
\end{array}\right], \\
C_{c}=\left[\begin{array}{ll}
5.326 & 70.68
\end{array}\right], D_{c}=[0.03323] .
\end{gathered}
$$

Figure 2 shows the estimate of the basin of attraction $S_{0}$ and trajectories of the system (50) with the controller gains (51) for several initial conditions $x_{0} \in S_{0}$. As observed is Remark 1 , the set $S_{0}$ is not invariant but for all $\xi(0) \in S_{0} \times\{0\}, \lim _{t \rightarrow \infty} \xi(t)=0$. This is illustrated in Figure 3 that shows the evolution of the Lyapunov function $V(\xi)=\xi(t)^{T} P \xi(t)$ and $V(x, 0)=x(t)^{T} Z x(t)$, that characterizes the set $S_{0}$, for the initial condition $\xi(0)=(-0.04,-0.3,0,0)$. We observe that $V(\xi)$ behaves as expected, whereas $V(x, 0)$ increases its value before converging to zero according to the trajectories presented in Figure 2 . 


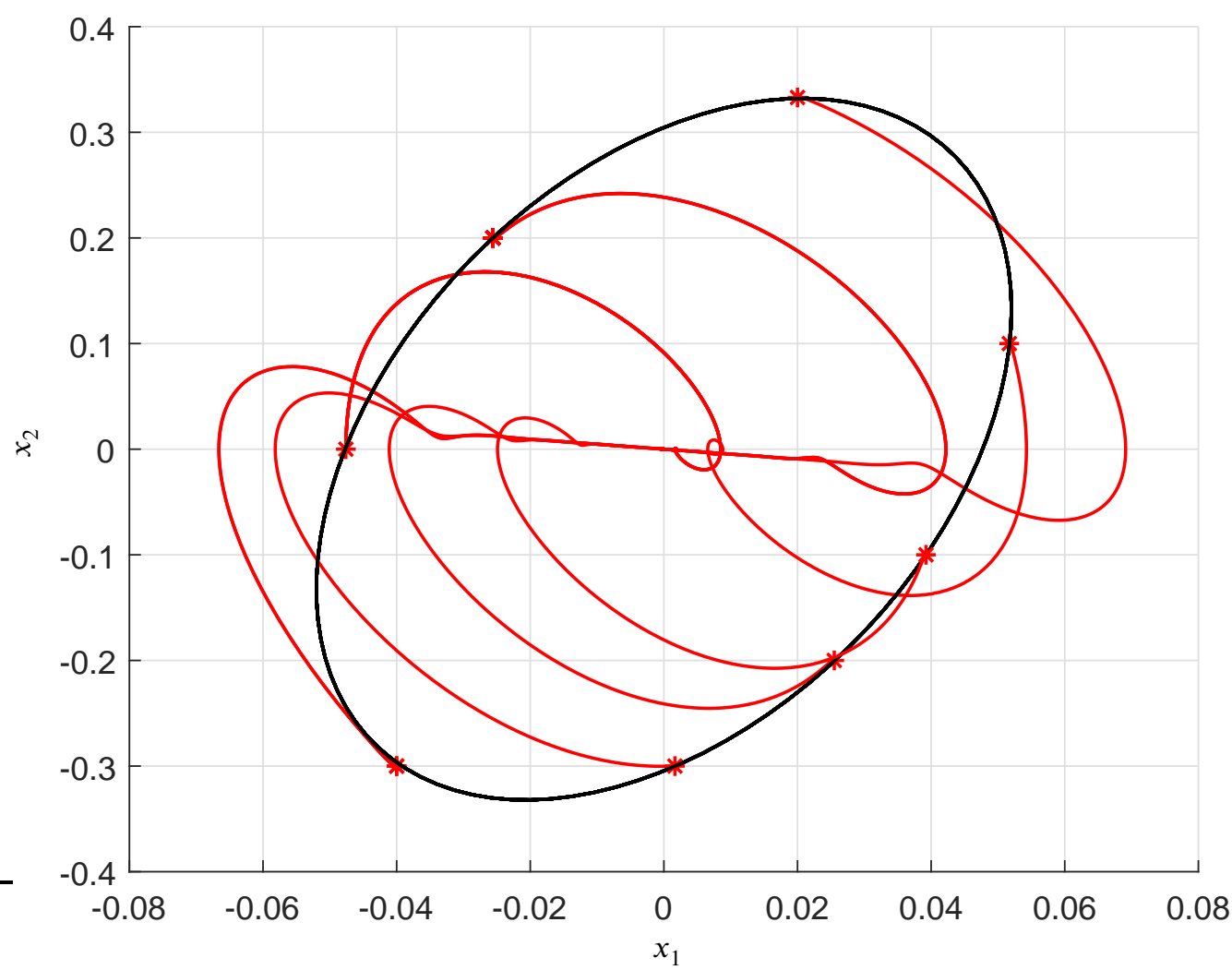

FIGURE 2 Estimation of the region of attraction $S_{0}$ and trajectories of the system (50) with the controller gains (51).

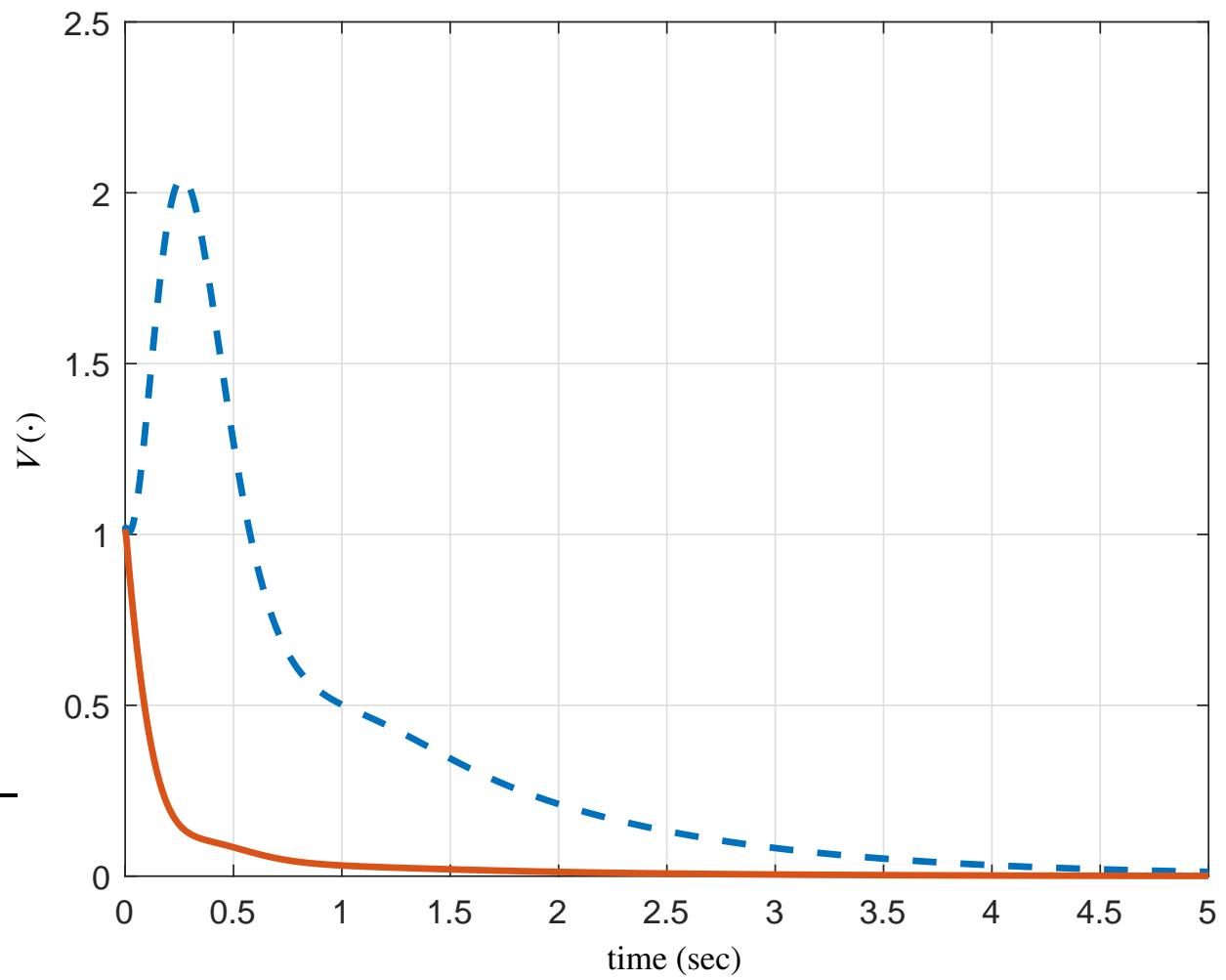

FIGURE 3 Trajectories of the Lyapunov function $V(\xi)=\xi(t)^{T} P \xi(t)$ (solid red line) and $V(x, 0)=x(t)^{T} Z x(t)$ (dashed blue line) for Example 2 with initial condition $\xi(0)=(-0.04,-0.3,0,0)$. 


\section{6 | CONCLUSION}

In this paper, convex conditions to synthesize linear and nonlinear full-order dynamic output feedback control laws to locally exponentially stabilize quadratic systems have been proposed. The design conditions are verified for the augmented state, gathering the states of the system and the controller, belonging to a level set of a Lyapunov function, the estimate of the basin of attraction of the origin. This procedure allows us to design the controller and to increase an estimate of the basin of attraction as large as possible at the same time in opposition to the polytopic approach, also a contribution of this work, where the enlargement of the polytopic region of the state-space and the controller design have to be performed alternately requiring heuristics or iterative algorithms. The numerical experiments show that we are able to compute larger estimates of the basin of attraction compared with the ones achieved with polytopic-based paradigm.

\section{References}

1. Bonnard B. Quadratic control systems. Mathematics of Control, Signals and Systems 1991; 4(2): 139-160.

2. Lotka AJ. Elements of Physical Biology. Williams \& Wilkins Company . 1925.

3. Volterra V. Fluctuations in the abundance of a species considered mathematically. Nature 1926; 118: 558-560.

4. Cosentino C, Salerno L, Passanti A, Merola A, Bates DG, Amato F. Structural Bistability of the GAL Regulatory Network and Characterization of its Domains of Attraction. Journal of Computational Biology 2012; 19(2): 148-162.

5. Jurdjevic V, Kupka I. Polynomial Control Systems. Mathematische Annalen 1985; 272: 361-368.

6. Frayman M. On the relationship between bilinear and quadratic systems. IEEE Transactions on Automatic Control 1975; 20(4): 567-568.

7. Reid WT. Riccati differential equations. Academic Press, New York . 1972.

8. Walcher S. Algebras and differential equations. Hadronic Press . 1991.

9. Isidori A. Nonlinear Control Systems. Springer. third ed. 1995.

10. Baillieul J. Controllability and observability of polynomial dynamical systems. Nonlinear Analysis: Theory, Methods \& Applications 1981; 5(5): 543-552.

11. Markus L. Quadratic differential equations and nonassociative algebras. In: Cesari L, LaSalle J, Lefschetz S., eds. Contributions to the Theory of Nonlinear Oscillations. V. Princeton University Press. 1960 (pp. 185-213).

12. Koditschek DE, Narendra KS. The Stability of Second-Order Quadratic Differential Equations. IEEE Transactions on Automatic Control 1982; 27(4): 783-798.

13. Genesio R, Tartaglia M, Vicino A. On the estimation of asymptotic stability regions: State of the art and new proposals. IEEE Transactions on Automatic Control 1985; 30: 747-755.

14. Chesi G, Tesi A, Vicino A. Computing optimal quadratic Lyapunov functions for polynomial nonlinear systems via LMIs. In: 15th IFAC World Congress. ; 2002; Barcelona, Spain: 43-48.

15. Amato F, Cosentino C, Merola A. On the region of attraction of nonlinear quadratic systems. Automatica 2007; 43(12): 2119-2123.

16. Tarbouriech S, Queinnec I, Calliero TR, Peres PLD. Control design for bilinear systems with a guaranteed region of stability: an LMI-based approach. In: Mediterranean Control Association. ; 2009; Thessaloniki, Greece: 809-814.

17. Valmórbida G, Tarbouriech S, Garcia G. State feedback design for input-saturating quadratic systems. Automatica 2010; 46(7): 1196-1202. 
18. Chesi G. Domain of attraction: Analysis and Control via SOS Programming. 415 of Lecture Notes in Control and Information Sciences. Springer-Verlag London . 2011.

19. Sadabadi MS, Peaucelle D. From static output feedback to structured robust static output feedback: A survey. Annual Reviews in Control 2016; 42: 11-26.

20. Yang X, Chen L, Burton R. Stability of discrete bilinear systems with output feedback. International Journal of Control 1989; 50(05): 2085-2092.

21. Amato F, Ariola M, Consentino C, Merola A. Output feedback control of nonlinear quadratic systems. In: Proceedings of the 49th IEEE Conference on Decision and Control ; 2010; Atlanta, GA, USA: 3349-3354.

22. Andrieu V, Tarbouriech S. Global Asymptotic Stabilization for a Class of Bilinear Systems by Hybrid Output Feedback. IEEE Transactions on Automatic Control 2013; 58(6): 1602-1608.

23. Quinn JP. Stabilization of Bilinear System by Quadratic Feedback Controls. Journal of Mathematical Analysis and Applications 1980; 75: 66-80.

24. Coutinho D, de Souza CE. Nonlinear state feedback design with a guaranteed stability domain for locally stabilizable unstable quadratic systems. IEEE Transactions on Circuits and Systems I: Regular Papers 2012; 59(2): 360-370.

25. Tognetti ES, Calliero TR, Jungers M. Output feedback control for bilinear systems: a polytopic approach. In: 3rd IFAC Workshop on Linear Parameter Varying Systems ; 2019; Eindhoven, Netherlands: 58-63.

26. Jelali M, Schwarz H. Volterra series expansion for state quadratic systems. International Journal of Applied Mathematics and Computer Science 1996; 6(1): 67-76.

27. Jelali M, Schwarz H. On the quadratic modeling of nonlinear plants with application to an electro-hydraulic drive. In: 3rd IFAC Symposition Nonlinear Control Systems Design. ; 1995; Tahoe City, California: 379-384.

28. Amato F, Cosentino C, Fiorillo AS, Merola A. Stabilization of Bilinear Systems Via Linear State-Feedback Control. IEEE Transactions on Circuits and Systems II: Express Briefs 2009; 56(1): 76-80.

29. de Oliveira MC, Skelton RE. Stability tests for constrained linear systems. In: Reza Moheimani SO., ed. Perspectives in Robust Control. 268 of Lecture Notes in Control and Information Science. New York, NY: Springer-Verlag. 2001 (pp. 241-257).

30. Scherer C, Gahinet P, Chilali M. Multiobjective output-feedback control via LMI optimization. IEEE Transactions on Automatic Control 1997; 42(7): 896-911.

31. Petersen IR. A stabilization algorithm for a class of uncertain linear systems. Systems \& Control Letters 1987; 8(4): 351-357.

32. Boyd S, El Ghaoui L, Feron E, Balakrishnan V. Linear Matrix Inequalities in System and Control Theory. Philadelphia, PA: SIAM Studies in Applied Mathematics . 1994.

How to cite this article: E. S. Tognetti, T. R. Calliero, and M. Jungers (2020), Output feedback control for quadratic systems: a Lyapunov function approach, Int J Robust Nonlinear Control, 2020;00:1-15. 